\title{
An Assessment of the Results of Controlled Fractional Distraction by Joshi's External Stabilization System in Club Foot
}

\author{
${ }^{1}$ Ashwani Sadana, ${ }^{2}$ Chandra Prakash Pal, ${ }^{3}$ Karuna Shankar Dinkar
}

\begin{abstract}
Club foot is amongst the most common of congenital deformities. Its incidence is 1 to 2 per 1000 live births. At birth, the diagnosis can be made by observing the foot for forefoot adduction, hindfoot inversion and equinus deformity. The study was conducted to evaluate the role of controlled fractional distraction in the management of neglected club feet by Joshi's external stabilization system (JESS). Total of 18 cases (22 feet) were studied, which were corrected by JESS. All cases were evaluated clinically, radiologically, podographically, and Pirani scoring system, both, before and after the correction. Severity of the deformities and clinical correction was assessed by Pirani score. All patients achieved good clinical results as per Pirani score, radiological evaluation showed that all subjects achieved the normal range of values.
\end{abstract}

Keywords: Club foot, Inversion, JESS, Pirani, Adduction, Neglected.

How to cite this article: Sadana A, Pal CP, Dinkar KS. An Assessment of the Results of Controlled Fractional Distraction by Joshi's External Stabilization System in Club Foot. J Foot Ankle Surg (Asia-Pacific) 2015;2(1):13-16.

Source of support: Nil

Conflict of interest: None

\section{INTRODUCTION}

Club foot is among the most common of congenital deformities congenital talipses equinovarus (CTEV) is a complex three-dimensional deformity having four components-equinus, varus, adduction and cavus. The deformity continues to post many difficulties in its management. It is a hereditary foot deformity. Its incidence is 1 to 2 per 1000 live births. ${ }^{1}$ At birth, the diagnosis can be made by observing the foot for midfoot adduction, hindfoot inversion and equinus deformity. Lehman (1980) and Sompii (1984) classified the clubfoot as nonrigid, rigid and teratologic variety. Our aim is to eliminate or if not

\footnotetext{
${ }^{1,2}$ Assistant Professor, ${ }^{3}$ Lecturer

${ }^{1-3}$ Department of Orthopedics, SN Medical College, Agra Uttar Pradesh, India
}

Corresponding Author: Karuna Shankar Dinkar, Lecturer Department of Orthopedics, SN Medical College, Agra, Uttar Pradesh, India, Phone: 05622642264, e-mail: drksdinkar27@ yahoo.in possible, to reduce all elements of the club foot deformity, hence, achieving a functional, pain free, normal looking plantigrade, mobile foot. ${ }^{1}$ The factors associated with the poor prognosis are female child, hereditary, late age of presentation, severity of deformity, rigidity of foot, associated cavus. ${ }^{2,3}$ Kite $^{4}$ rationalized the whole treatment of clubfoot by conservative means. Satisfactory results are obtained by Ponseti and SM Dey ${ }^{5}$ method of manipulation and serial casting. Percutaneous soft tissue release and tenotomy for getting the corrected foot had been advocated by various workers. ${ }^{7}$ The method of controlled differential distraction, ${ }^{5}$ that is, ligamentotaxis, along with the mini external fixator was originally described by Dr BB Joshi in 1990. Ilizarov fixator ${ }^{8}$ has also been used for correction of CTEV deformities. We considered any clubfoot presented first time to us for the management at or after the age of 1 year. This study was conducted to evaluate the clinicoradiological outcomes of neglected idiopathic CTEV managed by Joshi's External Stabilization System. We, in our study, intended to use the indigenous assembly of distracters and static rods held by link joints to transfixed K-wires for correction of all the components of this deformity. This method involves controlled differential fractional distraction to correct all the aspects of central deformity by gradual stretching of soft tissue. It has got some inherent merits over other prevelant technique. This is a semi-invasive technique. Length of the foot is effectively lengthened in contra distinction to other methods in which shortening is associated because of osteotomy or arthrodesis.

\section{MATERIALS AND METHODS}

This observational study was conducted on all the patients with late presentation of CTEV. Since all cases of talipes equino varus not amenable to correction by manipulation and plaster stretching are candidates for this method.

\section{Inclusion Criteria}

- Drop outs of conservative treatment.

- Neglected variety.

- Recurrence after surgical release. 
Patients below 3 years and above 6 years of age are excluded. We also excluded cases associated with secondary causes like arthrogryposis, meningomyelocele, and so forth.

Patients were thoroughly assessed clinically including podograms and radiologically. Radiological assessments were done in $\mathrm{AP}$ and lateral view in stress dorsiflexion in all cases. X-rays were studied for talocalcaneal angle, talo-first metatarsal angle, talo-Vth metatarsal angle (all in AP view), talocalcaneal angle, tibiocalcaneal angle and Calcaneal pitch (all in lateral view) was used in this study. Severity of deformity was assessed by using Pirani scoring system and to assess the correction achieved after final casting. Podograms were taken to assess the weight bearing portion of foot, length, and width of foot before and after completion of treatment.

All patients were operated in general anesthesia. Basic assembly consists of three sites of pin holds (Tibial, calcaneal, metatarsal) and three pairs of connections of which tibiocalcaneal and calcaneometatarsal were distractors and tibiometatarsal were connecting rods. After putting all the K-wires (i.e. 3 each tibial, calcaneal, and metatarsal), we tried to reduce the deformity by Ponseti method and then by connecting the tibial, calcaneal, and metatarsal attachments we tried to maintain the reduction. After that distracters were placed on both sides between tibial-calcaneal and calcaneal-metatarsal attachments (Figs 1A and B).

On the third postoperative day, distraction were started that is, $0.25 \mathrm{~mm} 6$ hourly on the medial side while $0.25 \mathrm{~mm} 12$ hourly on the lateral side in hospitalized patients. After discharge of the patients from hospital, their parents were instructed to do the distraction at the rate of $1 \mathrm{~mm}$ on medial side and $0.5 \mathrm{~mm}$ on lateral side once a day for convenience. The first phase ends after clinical and radiological correction of forefoot adduction. Visual correction of deformities were noted during the distraction phase. Weekly X-rays were taken to see correction while in distraction phase. Differential distraction on medial side is performed twice the rate than that of lateral side. Distraction on lateral side not only prevents crushing of the articular cartilage but also permits normal growth of epiphyseal plate on lateral side which may be affected if compression is done on the lateral side.

After this initial distraction phase of approximately 3 to 6 weeks the assembly were held in static position for further 3 weeks to allow soft tissue maturation in elongated position. After that assembly were removed and plaster cast were applied in a position of maximum correction. The children were allowed to ambulate full weight bearing in plaster. Three weeks later, more plaster cast were applied. After that appropriate orthosis and/ or splint were applied and patients were followed-up regularly. At the end of 6,12,18 and 24 months, all clinical assessments were done and documented. Radiological assessment was also done at the end of 1 year follow-up and was analyzed. After 24 months follow-up, patients were told to contact for follow-up annually. They were told to report in case of relapse of any deformity. Cases were considered as failure if:

- There was no or incomplete clinicoradiological correction or

- Complications like joint subluxation, rocker bottom deformity occurred.

\section{EVALUATION OF RESULTS}

Results were evaluated on the basis of pirani scoring system, podogram, radiologically and foot bimalleolar angle parameters. A final Pirani score of 0 to 2 is regarded as good clinical correction achieved. The radiographic examination is accomplished by taking anteroposterior and lateral radiographs. Talocalcaneal angle is taken in both anteroposterior and lateral view. The summation of a anteroposterior and lateral talocalcaneal angles is talocalcaneal index which has normal value of angle of 40 to $85^{\circ}$.

\section{OBSERVATION AND RESULTS}

In our study, we managed 22 feet of 18 cases out of which 11 feet of failed conservative management. Neglected

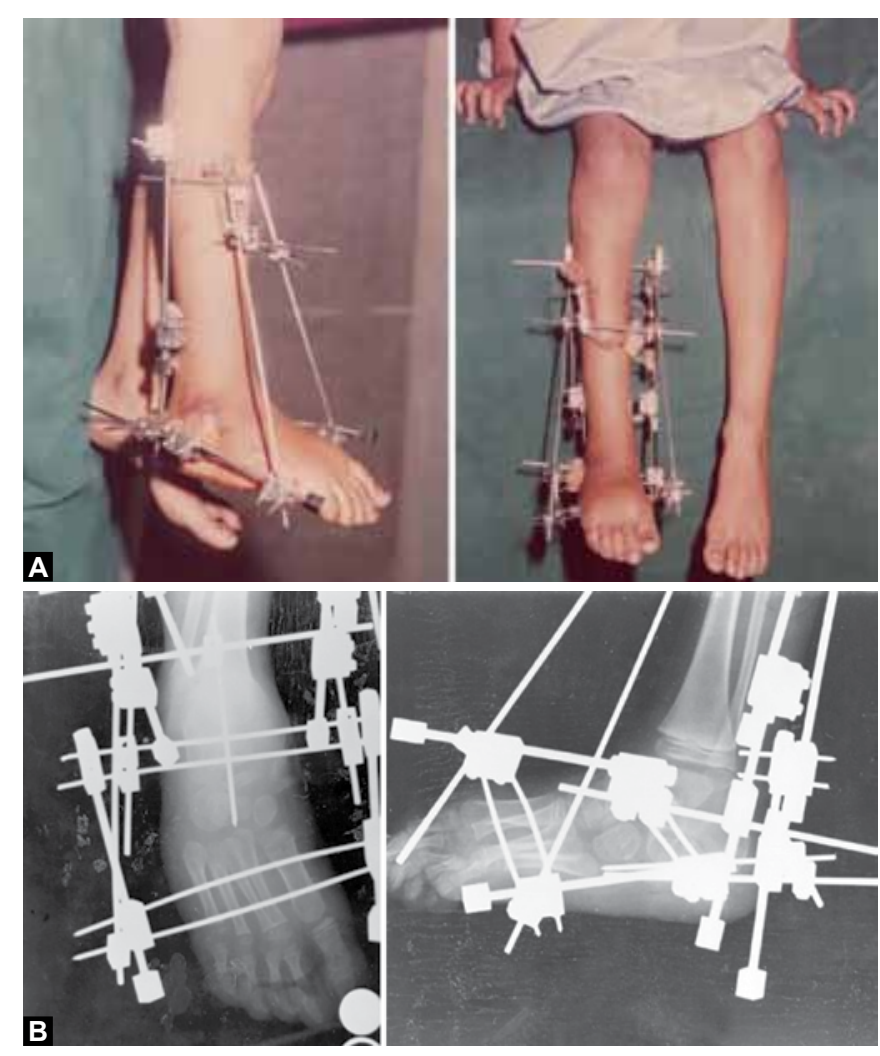

Figs $1 \mathrm{~A}$ and $\mathrm{B}$ : Clinical photograph at 3 weeks of distraction and $(B)$ radiograph at 4 weeks of distraction 
untreated feet were 9 in number and relapsed variety after surgical release were 2 feet. Majority of cases were in the age group of 2 to 6 years. There were 10 males and 8 females patients. Mean age of the patient was 4.05 years. Right side was more commonly affected in unilateral cases. Clinicoradiological parameters were also improved in all patients. Only 3 (13.62\%) feet developed superficial infection $4(18.16 \%)$ feet presented with relapsed forefoot adduction (corrected by manipulations and retention by plasters in all cases) and all returned to orthosis. No open correction of any component of deformity in any case at any stage was done. Talocalcaneal angle in anteroposterior and lateral view was in most cases between ranges of 26 and $38^{\circ}$ while talocalcaneal index was between the range of 52 and $74^{\circ}$.

The follow-up period in the study varied from 6 to 23 months and at end of follow-up excellent to good results were seen in 14 feet and fair results in 8 feet (36.32\%). Though the follow-up is short, we are pursuing these cases for longer follow-up (Fig. 2).

To evaluate our end results, the subjects were graded on a scale of good to poor using Pirani Score. A final Pirani score of 0 to 2 is regarded as good clinical correction achieved. All patients were reverted to 0 to 2 group, that is, good outcome. Before correction the mean Pirani Total score was 5 , which was reduced to 0.7 after the correction, that is, all became more flexible than earlier.
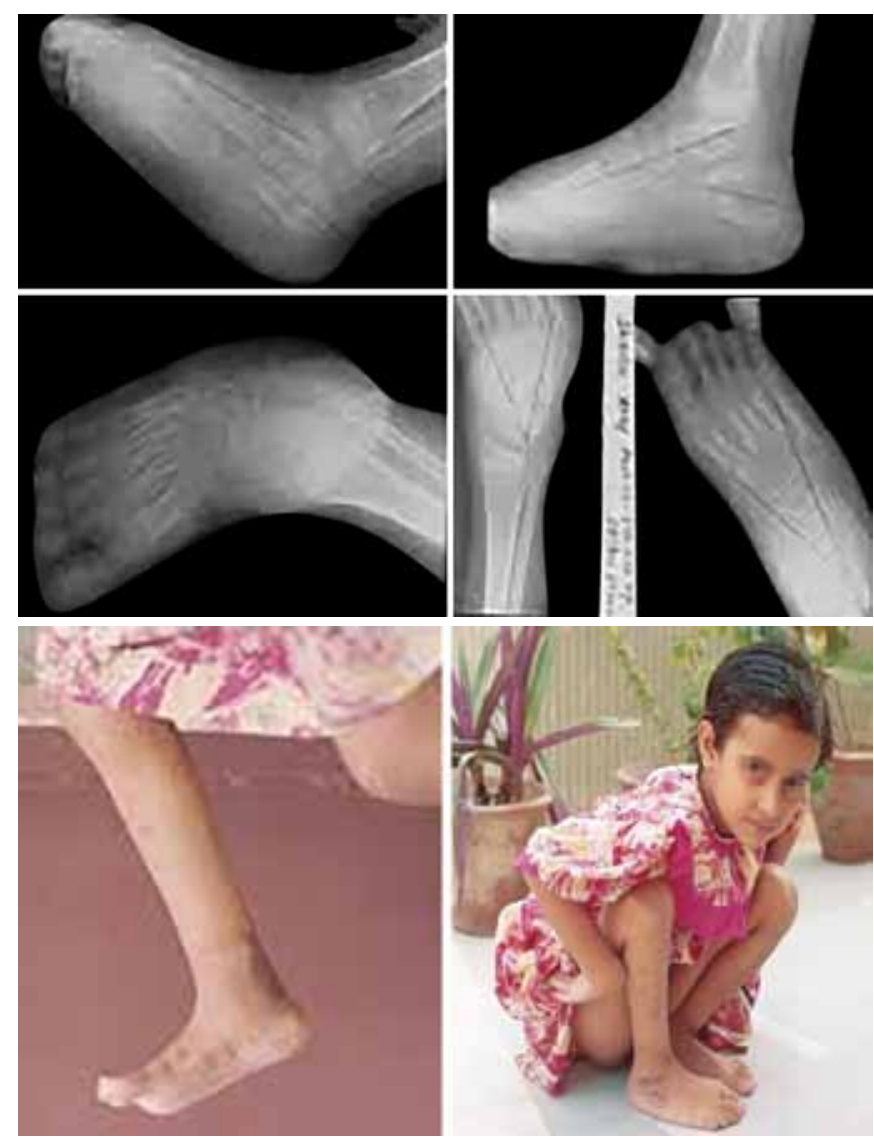

Fig. 2: Radiological and follow-up of patient
By the end of the follow-up, the flexibility of the feet remained unchanged.

\section{DISCUSSION}

Congenital talipes equinovarus is a common orthopedic problem in children, which have a bulk of the congenital anomalies. Various treatment options including conservative treatment by Ponseti ${ }^{2-6,9,11,12}$ have been given by various authors for management of club foot with variable success rate. Surgery is mainly advocated for late, neglected, and relapsed feet yet many of them including Lehman et al have stated that so called resistant variety of club foot can usually be diagnosed even on first examination of child. These children have a short heel and less pliable foot. These resistant variety always needs some form of operative intervention.

Manipulation and correction by Ponseti technique had been accepted by many orthopedic surgeons as method of choice. Early correction can be achieved with a low recurrence rate by this method.

In our study, we excluded patients which were below the age of 3 years because there was a risk that their soft bones may not be able to bear the distraction forces. We have opinion that child below the age of 3 years can be managed by nonoperative method like Ponseti or by soft tissue procedures. Children which were above 6 years of age were also excluded as at this age group there are significant bony changes which may affect the outcome of study.

We managed these feet by differential distraction by JESS. ${ }^{6}$ It is a semi-invasive procedure, as it does not require any open or percutaneous surgical procedure for the deformity correction. When we desired clinical correction were achieved, foots were supported in corrected position by above knee plaster cast for next 4 to 6 weeks and then were on DB splint. The major drawback was acceptance of assembly by the children. There was also chance of injuries to the children and their attendants while nursing. But the results are quite encouraging giving good correction in much short period. In our study, improvement in medial and lateral border ratio was observed in all cases but complete reversal of ratio could not be obtained. In unilateral cases affected foot remained smaller than normal foot but was cosmetically acceptable.

Jain et $\mathrm{al}^{10}$ in their study FBA parameter showed improvement from grade 3 to grade 1 in $93 \%$ cases but our study this parameter improved $82 \%$ from grade 3 to grade 1 .

In our study, radiological parameters were returned to near normal range. The explanation may be that the primary pathology in CTEV is of soft tissue contractures in 
midfoot and hindfoot. Bony articulation are not marked as skeleton is mainly cartilaginous. So, the main role of distraction is to stretch the contracted ligaments gradually and differentially.

In a study on 34 cases of severe and neglected cases treated by Ilizarov fixator, give good results in about $58.8 \%$ cases with recurrence in $8.7 \%$ cases. While a study on 44 cases of neglected cases of CTEV which were treated by JESS and followed for about 2 years give $90 \%$ excellent to good results.

Joshi's External Stabilization System assembly differs from the Ilizarov technique by the following:

- In JESS, axially tensioned wires are not used.

- Club foot is a multiplanar, multiapical deformity so it is very difficult to plan the location of an external hinge for deformity correction. Our frame is unconstrained and relies on correction occurring at the natural joints.

- In JESS, deformity is corrected by differential distraction so that deformity is corrected without compressing the child's foot. Due to which articular cartilage is not damaged and there is normal growth of epiphyseal plate.

- The Ilizarov ring frame is very complex and is not suited for pediatric foot while JESS, frame is light and versatile.

Though, it is a very small series but by far we are able to achieve very encouraging and comparable results.

\section{CONCLUSION}

We may conclude from the study that correction of late presented CTEV by ligamentotaxis is patient's and surgeon friendly procedure. But in this procedure, the active participation of the patients' attendants is one of the prime factors for the successful outcome.

\section{REFERENCES}

1. Ikeda K. Conservative treatment of idiopathic clubfoot. J Pediat Orthopaed 1992;12(2):217-223.

2. Göksan SB. Treatment of congenital clubfoot with the ponseti method. Acta Orthopaedica et Traumatologica Turcica 2002;36(4):281-287.

3. McKay DW. New concept of and approach to clubfoot treatment: section II-correction of the clubfoot. J Pediat Orthopaed 1983;3(1):10-21.

4. Kite JH. Principles involved in the treatment of congenital clubfoot. J Bone Joint Surg 1939;21(3):122-129.

5. Ponseti IV, Smoley EN. Congenital clubfoot-the results of treatment. J Bone Joint Surg 1963;45(2):134-141.

6. Marthya HA, Arun B. Short term results of correction of CTEV with JESS distractor. J Orthopaed 2004;1(1):e3.

7. Turco VJ. Surgical correction of the resistant club foot. One-stage posteromedial release with internal fixation: a preliminary report. J Bone Joint Surg 1971;53(3):477-497.

8. Correll J, Forth A. Correction of severe clubfoot by Ilizarov method. J Foot Ankle Surg 2003;2(1):27-32.

9. Suresh S. Management of late presented severe club foot. J Orthopaed Surg 2003;11(2):194-201.

10. Jain AK, Zulfiqar AM, Kumar S, Dhammi IK. Evaluation of foot bimalleolar angle in the management of congenital talipes equinovarus. J Pediat Orthopaed 2001;21(1):55-59.

11. Colburn M, Williams M. Evaluation of the treatment of idiopathic clubfoot by using the Ponseti method. J Foot Ankle Surg 2003 Sep-Oct; 42(5):259-267.

12. Jowett CR, Morcuende JA, Ramachandran M. Management of congenital talipes equinovarus using the Ponseti method: a systematic review. J Bone Joint Surg Br 2011 Sep;93(9):1160-1164. 